\title{
Placas anteriores são mais efetivas do que parafusos iliossacrais na fixação da articulação sacroilíaca? Estudo Biomecânico*
}

\section{Are Anterior Plates More Effective than Iliosacral Screws to Fix the Sacroiliac Joint? Biomechanical Study}

\author{
${ }^{1}$ Departamento de Ortopedia, Américas Medical City, Rio de Janeiro, \\ RJ, Brasil \\ 2 Laboratório de Biomateriais em Ortopedia, Escola de Ciências \\ Médicas, Universidade de Campinas, Campinas, SP, Brasil \\ ${ }^{3}$ Departamento de Ortopedia, Escola de Ciências Médicas, \\ Universidade de Campinas, Campinas, SP, Brasil
}

Flavio Goldsztajn ${ }^{1}$ Jose Ricardo L. Mariolani2(- William Dias Belangero ${ }^{2,3(0)}$

\begin{abstract}
Endereço para correspondência Flavio Goldsztajn, Orthopaedic Trauma Surgeon, Serviço de Ortopedia e Traumatologia -Americas Medical City, Av Jorge Curi 550, Barra da Tijuca, Rio de Janeiro, RJ, 22.775-001, Brasil (e-mail: fgold@terra.com.br).
\end{abstract}

Rev Bras Ortop 2020;55(4):497-503.

\section{Resumo \\ Palavras chave \\ - placas ósseas \\ - instabilidade articular \\ - articulação sacroilíaca \\ - fenômenos biomecânicos \\ Introdução Usualmente, as luxações sacroilíacas são tratadas com parafusos iliossa- crais ou com placas anteriores à articulação sacroilíaca (ASI). Este estudo compara a rigidez e carga máxima suportada pelos dois tipos de fixações acima citados, utilizando pelves sintéticas. \\ Método Dez pelves sintéticas foram divididas em dois grupos $(n=5)$. No grupo denominado PlaCF, a ASI foi fixada com duas placas anteriores. No grupo ParCF, a ASI foi fixada com dois parafusos iliossacrais no corpo da primeira vertebra sacral (S1). A rigidez e carga máxima suportada por cada montagem realizada, foi mensurada. A análise estatística foi realizada através do teste $U$ de Mann-Whitney $(p<0.05$ foi considerado estatisticamente significativo para todas as análises). \\ Resultados A carga máxima suportada até a falha da fixação pelos grupos PlaCF e ParCF foram respectivamente $940 \pm 75 \mathrm{~N}$ e $902 \pm 56 \mathrm{~N}$, não havendo diferença esta- tística entre eles. A rigidez obtida pelo grupo ParCF foi maior e com diferença estatística em relação ao grupo PlaCF (68.6 $\pm 11.1 \mathrm{~N} / \mathrm{mm}$ e $50 \pm 4.0 \mathrm{~N} / \mathrm{mm}$ respectivamente). \\ Conclusão Apesar da menor rigidez obtida no grupo PlaCF, as placas anteriores à ASI podem ser uma ótima opção no tratamento da luxação sacroilíaca quando os parafusos iliossacrais não puderem ser utilizados. Outros estudos são necessários para detectar possíveis diferenças entre os dois procedimentos do ponto vista cirúrgico e clínico.}

Trabalho realizado no Laboratório de Biomateriais em Ortopedia, Escola de Ciências Médicas, Universidade de Campinas, Campinas, SP, Brasil. recebido

22 de Fevereiro de 2019

aceito

18 de Julho de 2019
DOI https://doi.org/

10.1055/s-0039-1697973. ISSN 0102-3616.
Copyright $\odot 2020$ by Sociedade Brasileira License terms de Ortopedia e Traumatologia. Published by Thieme Revinter Publicações Ltda, Rio de Janeiro, Brazil 


\begin{abstract}
Introduction Sacroiliac joint dislocations are caused by high energy trauma and commonly treated with the iliosacral screw fixation or the anterior plating of the sacroiliac joint (SIJ). However, there is a lack of consensus regarding which procedure is the most successful in treating sacroiliac joint dislocations. This aims to compare stiffness and maximum load of pelvises with sacroiliac joint dislocations treated with both procedures in a synthetic bone model.

Methods Synthetic pelvises were mounted and divided into 2 treatment groups $(n=5)$ : a model with two orthogonal plates placed anteriorly to the SIJ (PlaCF group) and another with two iliosacral screws fixating the SIJ (ParCF group), both with pubic symphysis fixation. The maximum load supported by each sample was observed and the stiffness was calculated from the curve load vs displacement. The mean values of load to failure and stiffness for each group were compared with the Mann-Whitney $\mathrm{U}$ test ( $p<0.05$ was considered significant for all analysis).

Results The mean load to failure supported by the PlaCF group was $940 \pm 75 \mathrm{~N}$ and the ParCF was $902 \pm 56 \mathrm{~N}$, with no statistical difference. The ParCF group showed higher values of stiffness $(68.6 \pm 11.1 \mathrm{~N} / \mathrm{mm})$ with statistical significant difference in

\section{Keywords}

- bone plates

- joint instability

- sacroiliac joint

- biomechanical phenomena

comparison to the PlaCF sample $(50 \pm 4.0 \mathrm{~N} / \mathrm{mm})$. The mode of failure was different in each group tested.

Conclusion Despite lower stiffness, the anterior plating fixation of the sacroiliac joint can be very useful when the iliosacral screw fixation cannot be performed. Further studies are necessary to observe any differences between these two procedures on the clinical and surgical setting.
\end{abstract}

\section{Introdução}

Luxações da articulação sacroilíaca (ASI) são geralmente decorrentes de traumas de alta energia. As lesões pélvicas podem danificar as estruturas intrapélvicas e o feixe neurovascular, produzindo hemorragias graves com altas taxas de mortalidade. $^{1-4}$

Uma vez que a ASI contribui significativamente para a estabilização do anel pélvico, suas fraturas e/ou luxações são desafiadoras do ponto de vista cirúrgico. Caso não tratadas de maneira adequada, essas lesões podem provocar dor, encurtamento dos membros e danos neurológicos, bem como disfunções sexuais e urinárias após o procedimento. ${ }^{2-4}$

Os maus resultados estão diretamente relacionados à fixação inadequada da lesão pélvica, que pode causar disfunção a longo prazo, devido à falha na osteossíntese e perda da redução da ASI. Por proporcionar boa estabilidade à articulação e ser minimamente invasiva, a fixação da ASI com parafusos iliossacrais em S1 tem sido amplamente utilizada. ${ }^{5,6}$ No entanto, esse procedimento requer imagens intraoperatórias adequadas, nem sempre possíveis de serem realizadas. Além disso, o dismorfismo do sacro pode dificultar ou mesmo impedir a inserção de parafusos iliossacrais no corpo de $\mathrm{S} 1$. Nestes casos, os riscos de complicações são altos, em especial em pacientes com anomalias sacrais ou que não obtiveram uma redução anatômica da articulação. ${ }^{7}$

Nessas situações, a redução aberta através da janela lateral da abordagem ilioinguinal é uma opção e, assim, fixação interna da ASI com duas placas ortogonais anteriores passa a ser uma boa alternativa aos parafusos iliossacrais. ${ }^{8,9}$
Este estudo compara o comportamento mecânico de parafusos iliossacrais e duas placas anteriores. Neste trabalho realizou-se uma avaliação biomecânica, in vitro, dessas duas formas de fixação da ASI. Comparou-se a carga máxima suportada por cada uma destas duas montagens, assim como a rigidez e o mecanismo de falha de cada uma delas.

\section{Material e Método}

Neste estudo foram utilizadas pelves sintéticas da marca numero 4060(Synbone AG, Zizers, Suíça) com a sínfise pubiana aberta.

Foi reproduzida a lesão tipo $\mathrm{C}$ de Tile. Dez pelves sintéticas foram divididas em dois grupos $(n=5)$. Dois diferentes modelos fixando a ASI foram testados: dois parafusos iliossacrais (ParCF) e duas placas ortogonais colocadas no aspecto anterior da ASI (PlaCF). Em ambos os grupos, a sínfise pubiana foi fixada (-Figuras 1 e 2). Todos os testes foram realizados na ASI direita. Este estudo biomecânico não envolveu seres humanos e foi liberado de avaliação pelo comitê de ética da Universidade.

\section{Preparação dos Corpos de Prova}

Modelo PlaCF $(n=5)$ : Duas placas anteriores, na articulação sacroilíaca direita. As placas DCP 3,5 mm (Depuy-Synthes Companies, Raynham, MA, USA) de 3 furos foram fixadas anteriormente à articulação sacroilíaca com 2 parafusos fixos ao ilíaco e um parafuso no sacro, evitando os foramens sacrais. ${ }^{10}$

Modelo ParCF $(n=5)$ : Dois parafusos canulados, $7.0 \mathrm{~mm} \mathrm{X}$ $75 \mathrm{~mm}$ (Depuy-Synthes Companies), fixando o ilíaco ao 


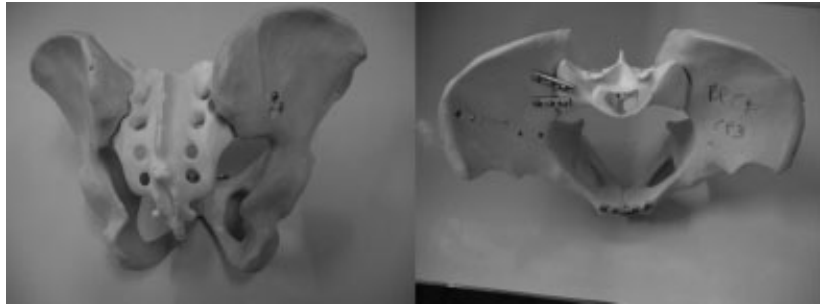

Figs. 1 e 2 Modelos sintéticos testados. Modelo com dois parafusos iliossacrais e sínfise pubiana fixada (ParCF) e modelo com duas placas anteriores à articulação sacroilíaca e sínfise pubiana fixada (PlaCF) respectivamente.

corpo de S1, respeitando o corredor de segurança da articulação sacroilíaca. 0 ponto de entrada do parafuso no ilíaco seguiu um trajeto perpendicular à articulação sacroilíaca ancorando-se no corpo de S1 sem violar anterior, posterior ou superiormente o corpo vertebral assim como o forâmen sacral, conforme descrito por Rout et al. ${ }^{11}$ Para confirmar a colocação dos parafusos no corredor de segurança e evitar discrepâncias na fixação das pelves sem imagem radiológica de controle, perfurou-se com o fio guia os limites do corpo vertebral de S1, para que todos saíssem no mesmo local. Após confirmação da posição adequada do fio guia, este foi recuado, a ponto de não ultrapassar os limites acima citados, e então foi feita a perfuração e fixação com o parafuso canulado.

A sínfise pubiana foi fixada com uma placa DCP 3,5mm de quatro furos, com dois parafusos corticais de cada lado da sínfise (Depuy-Synthes, J\&J company, USA).

Os orifícios para a introdução dos dispositivos de osteossíntese foram marcados previamente com caneta de tinta indelével para que não houvesse discrepância entre os modelos testados e todas as placas, parafusos corticais e parafusos canulados utilizados tinham as mesmas dimensões. Todos os parafusos foram introduzidos com o auxílio de um torquímetro, ajustado para um torque máximo de $1 \mathrm{Nm}$.

As pelves artificiais utilizadas possuíam, bilateralmente, um parafuso comum ligando o ilíaco ao sacro. Esse parafuso foi removido no lado direito de todos os modelos testados.

\section{Testes Biomecânicos e Análise Estatística}

Os ensaios foram realizados em uma máquina universal de ensaios EMIC DL3000 (Instron Brasil Equipamentos Científicos LTDA, São Jose dos Pinhais, PR, Brasil) acoplada a um computador, com o uso de uma célula de carga de $5.000 \mathrm{~N}$. As pelves sintéticas foram montadas com a hemipelve direita apoiada em uma prótese bipolar com cabeça de $42 \mathrm{~mm}$, fixada excentricamente ao cabeçote fixo (inferior) da máquina por meio de uma garra, de forma a simular a posição da marcha em que a carga sobre a cabeça do fêmur é máxima ${ }^{12}$ ( - Figura 3 ).

As pelves foram acopladas à célula de carga por meio de um dispositivo especialmente construído para manter o sacro na posição de uma pessoa ereta. Esse dispositivo consistia de uma peça de poliamida 12 confeccionada por impressão 3D, a qual, em sua parte inferior, reproduzia a geometria da região proximal do sacro e ali se encaixava, enquanto que sua parte superior encaixava-se em um aco-

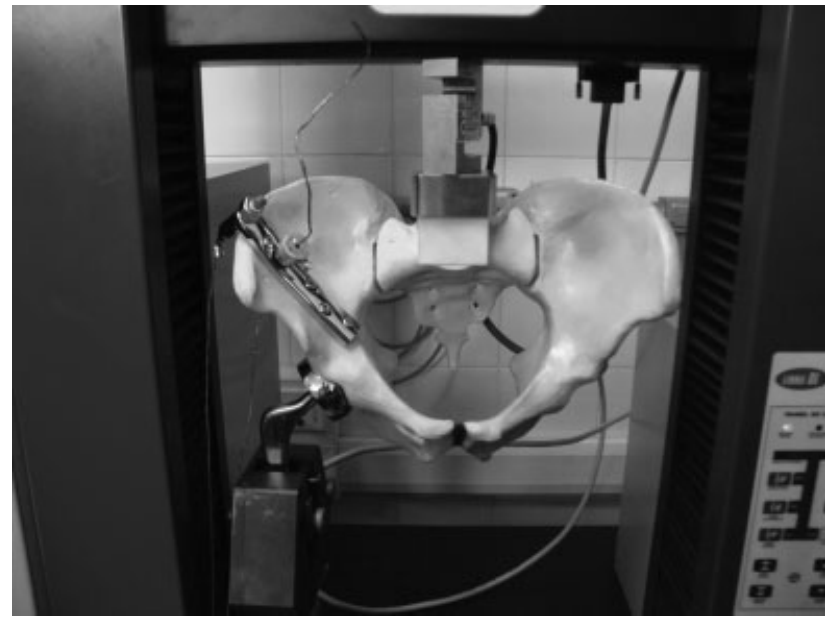

Fig. 3 Modelo pélvico sem nenhuma fixação mostrando como a pelve foi posicionada na máquina de teste.

plamento de aço conectado à célula de carga. Esta, por sua vez, era fixada ao cabeçote móvel (superior) da máquina de ensaio.

O ilíaco da hemipelve direita ainda foi conectado ao cabeçote inferior da máquina por meio de dois cabos de aço de $1,6 \mathrm{~mm}$ de diâmetro substituindo a musculatura glútea para estabilização da pelve. Os cabos foram fixados ao ilíaco com o auxilio de uma placa. Um dos cabos foi fixado à altura da crista ilíaca, e o outro sobre a linha glútea anterior, nas proximidades do tubérculo da crista ilíaca (-Figura 4).

Foi aplicada carga vertical de compressão ao modelo a uma velocidade de deformação de $5,0 \mathrm{~mm} / \mathrm{min}$. A força medida pela célula de carga e o deslocamento vertical do cabeçote superior medido por um sensor interno da máquina foram registrados pelo software Tesc ${ }^{\circledR} 3.04$ (Instron Brasil Equipamentos Científicos LTDA), que gerava em tempo real um gráfico de força versus deslocamento e salvava os dados para posterior análise. $O$ ensaio era interrompido automaticamente quando houvesse rompimento abrupto de algum componente do modelo ou manualmente quando o deslocamento vertical atingia aproximadamente $25 \mathrm{~mm}$.

De cada ensaio registrou-se a força máxima atingida e verificou-se visualmente o mecanismo de falha. A rigidez de cada montagem foi obtida a partir das curvas geradas pelo software da máquina, isolando-se a sua região linear e calculando-se a sua inclinação.

Foi utilizado o software Openstat para análise estatística. Os médias dos resultados de força máxima e rigidez obtidas por cada grupo foram comparadas com o uso do teste U de MannWhitney. Foi utilizado este modelo de análise estatística não paramétrica em virtude do tamanho da amostragem ser pequeno e de terem sido analisadas variáveis qualitativas. $p<0,05$ foi considerado significante para todas as análises.

\section{Resultados}

Foram testadas a força máxima suportada por cada montagem, assim como a capacidade de deformação (rigidez) de cada sistema. 


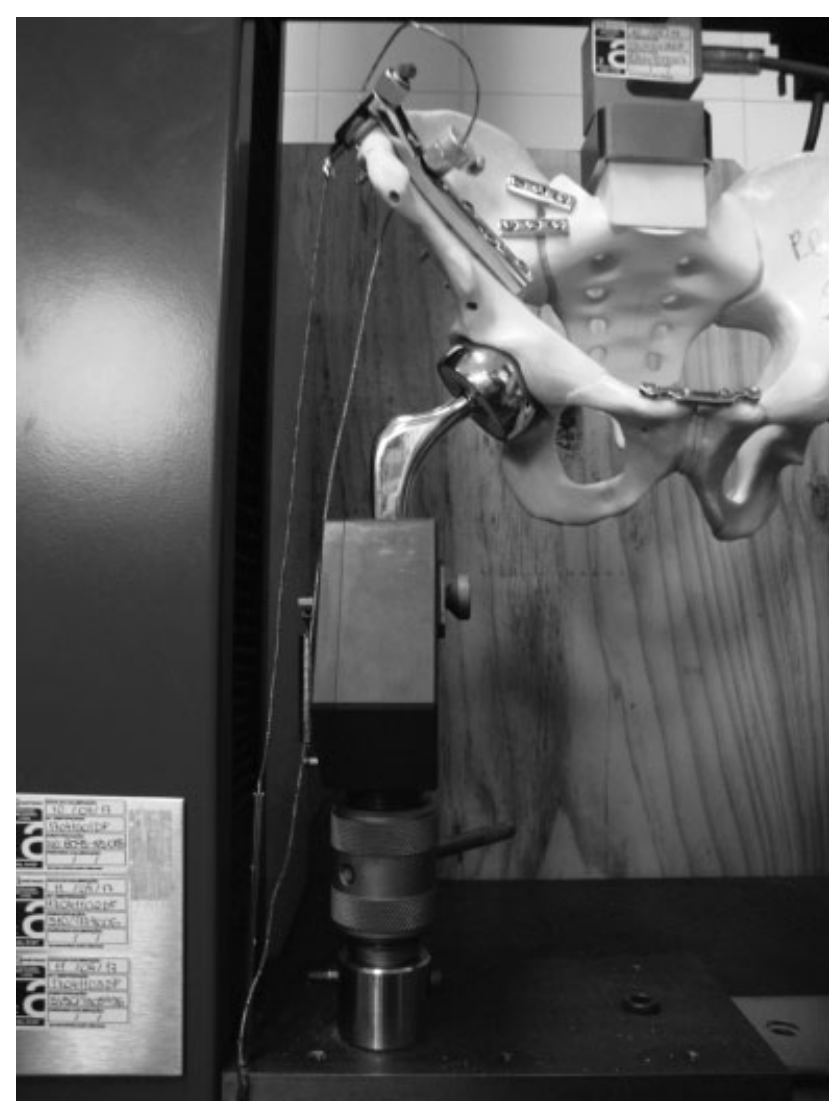

Fig. 4 O ilíaco da hemipelve direita ainda foi conectado ao cabeçote inferior da máquina por meio de dois cabos de aço de 1,6 mm de diâmetro substituindo a musculatura glútea para estabilização da pelve. Os cabos foram fixados ao ilíaco com o auxilio de uma placa. Um dos cabos foi fixado à altura da crista ilíaca, e o outro sobre a linha glútea anterior, nas proximidades do tubérculo da crista ilíaca. (fonte: arquivo pessoal).

Em relação a força máxima necessária para rompimento de cada montagem, o resultado final teve a seguinte média para os diferentes grupos testados:

PlaCF: 939,766 N com desvio padrão de 74,31

ParCF: 901,482 N com desvio padrão de 55,97

Em relação a rigidez de cada sistema, os diferentes grupos obtiveram as seguintes médias:

PlaCF: 49,924 N/mm com desvio padrão de 3,94

ParCF: 68,585 N/mm com desvio padrão de 11,06

Quando levada em consideração a força máxima exercida entre os grupos PlaCF e ParCF, não houve diferença estatística. Diferentemente, quando foi analisada a capacidade de deformação (rigidez) destes dois diferentes grupos, houve diferença estatística, com maior rigidez do grupo ParCF

As figuras 5 e $\mathbf{6}$ mostram respectivamente os resultados dos valores médios de carga máxima suportada e rigidez de cada modelo testado. A tabela 1 apresenta os valores médios e desvio padrão da carga máxima suportada e rigidez dos modelos testados, com a respectiva análise estatística. 0 teste U de Mann-Whitney não apontou diferença estatística em relação a carga máxima suportada pelos grupos PlaCF e $\operatorname{ParCF}(\mathrm{U}=17, \mathrm{z}=0,1481, \mathrm{p}>0,05$, bicaudal). Entretanto,

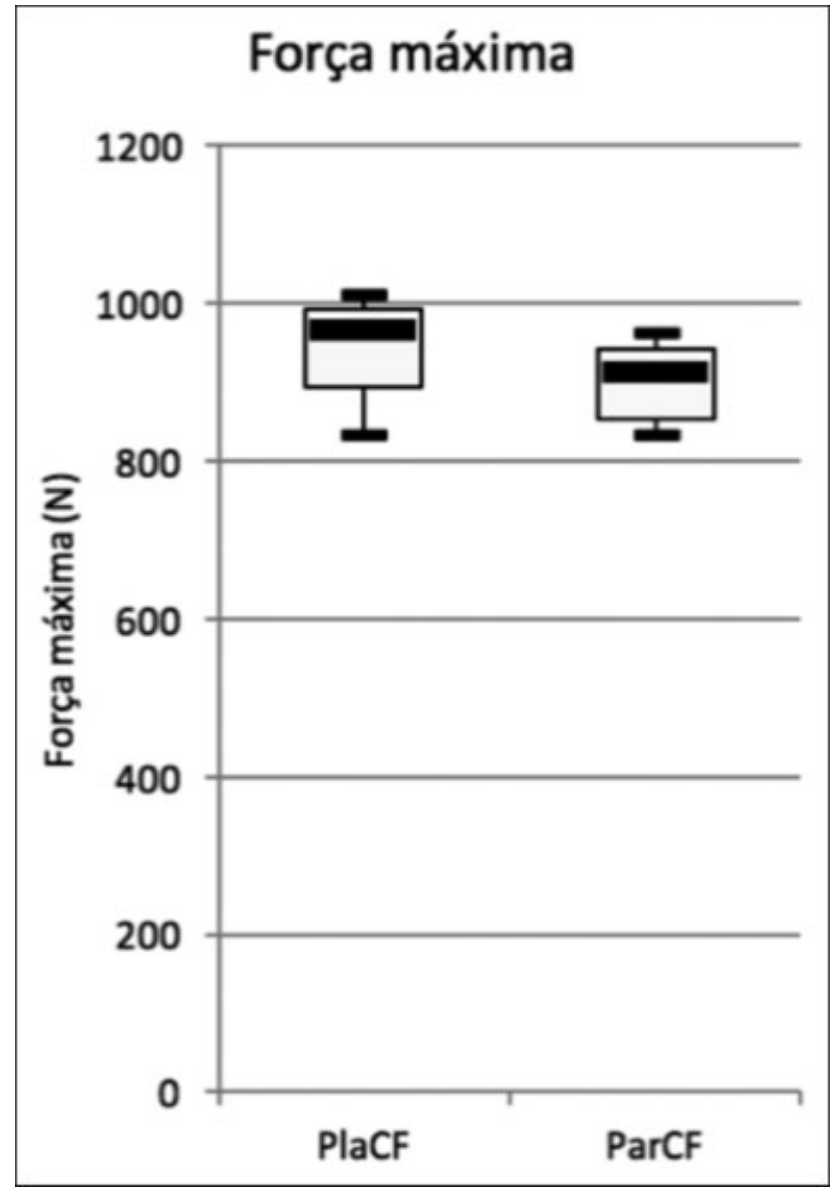

Fig. 5 Gráfico box-plot mostrando os valores médios de carga máxima suportada (N). Não existiu diferença estatística entre os grupos ParCF (dois parafusos iliossacrais em S1 fixando a articulação sacroilíaca com a sínfise pubiana fixada) e PlaCF (duas placas ortogonais fixadas anteriormente à articulação sacroilíaca com a sínfise pubiana fixada).

houve diferença estatística em relação a rigidez dos grupos testados ( $U=0, \mathrm{z}=0,0033, p<0,05$, bicaudal) .

Em relação ao mecanismo de falha, o comportamento foi diferente nos modelos avaliados. No grupo PlaCF houve arrancamento dos parafusos da placa (no ilíaco) na articulação sacroilíaca testada. No grupo ParCF, a falha ocorreu na articulação sacroilíaca contralateral. Os mecanismos de falha são mostrados na - Figura 7.

\section{Discussão}

As luxações sacroilíacas são lesões graves. Seu prognóstico está diretamente relacionado com a obtenção de uma redução anatômica articular e com a estabilidade obtida no ato cirúrgico. ${ }^{13,14} \mathrm{~A}$ utilização de parafusos iliossacrais tem se mostrado um excelente método de tratamento na luxação sacroilíaca. É uma técnica minimamente invasiva, com menor perda sanguínea e com menor risco de infecção. É necessário, entretanto, curva longa de aprendizado. ${ }^{15}$ Além disso, pode haver dificuldade na obtenção de imagens radiológicas adequadas intraoperatoriamente em virtude da presença de contraste intra-abdominal, gases intestinais 


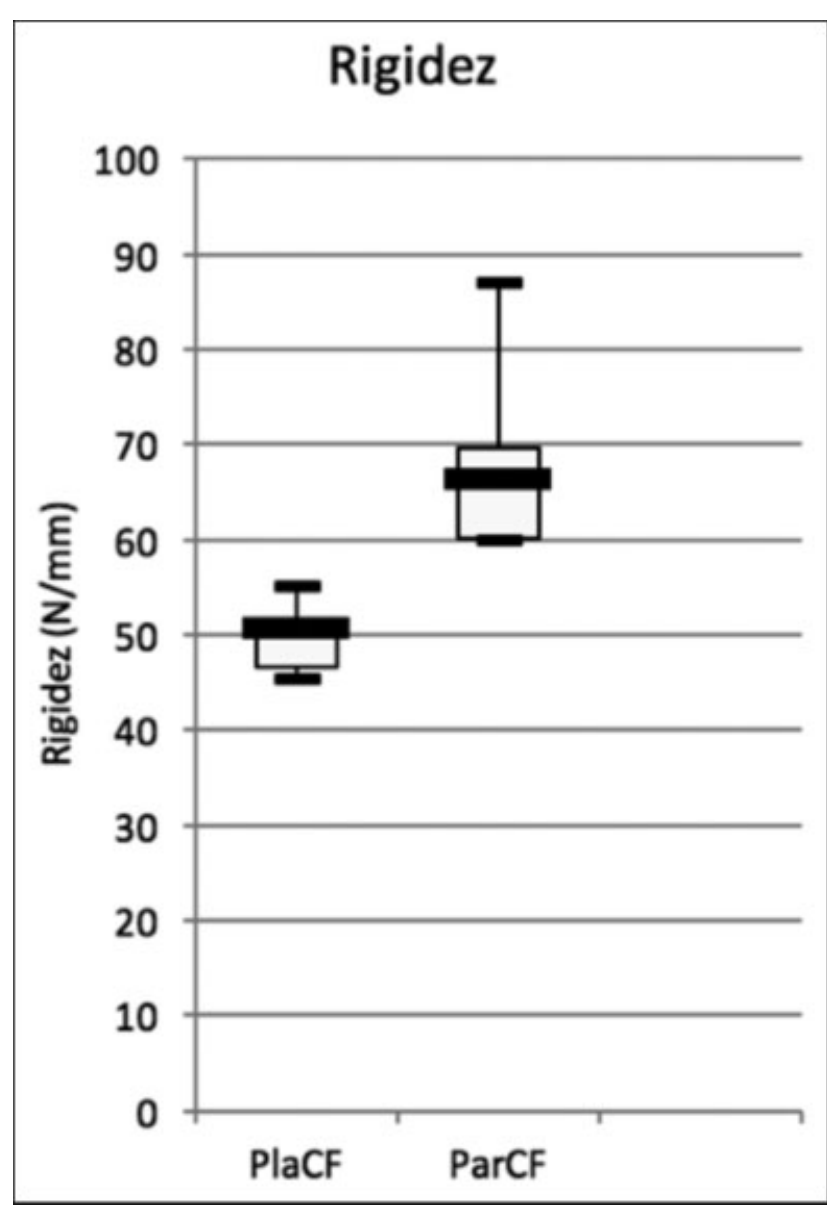

Fig. 6 Gráfico box-plot mostrando os resultados para rigidez de cada grupo testado $(\mathrm{N} / \mathrm{mm})$. O teste $U$ de Mann-Whitney apontou significativa diferença estatística entre ParCF (dois parafusos iliossacrais em S1 fixando a articulação sacroilíaca com a sínfise pubiana fixada) e $\mathrm{PlaCF}$ (duas placas ortogonais fixadas anteriormente à articulação sacroilíaca com a sínfise pubiana fixada).

ou obesidade mórbida. ${ }^{16}$ Variação anatômicas do sacro, tal como dismorfismo, ${ }^{7}$ pode tornar este procedimento extremamente difícil de ser realizado, aumentando assim o risco de complicações. Nas situações citadas acima, uma alternativa para o tratamento da luxação sacroilíaca seria a fixação com duas placas anteriores à articulação. Existem relatos na literatura em relação a lesões iatrogênicas durante abordagem anterior à articulação sacroilíaca. ${ }^{17}$ Salama et al. ${ }^{18}$ demonstraram excelentes resultados com taxas de complicações aceitáveis, utilizando esta técnica.

Tabela 1 Valores médios de carga máxima suportada por cada grupo de teste e sua respectiva rigidez ( \pm desvio padrão) com o resultado da análise estatística comparativa entre eles

\begin{tabular}{|l|l|l|l|l|}
\hline Parãmetro & Modelo & $\begin{array}{l}\text { Valor } \\
\text { médio }\end{array}$ & $\begin{array}{l}\text { Desvio } \\
\text { padrão }\end{array}$ & $p$ \\
\hline $\begin{array}{l}\text { Carga média } \\
\text { suportada (N) }\end{array}$ & PlaCF & 940 & 75 & \multirow{2}{*}{$>0.05$} \\
\cline { 2 - 4 } & ParCF & 902 & 56 & \\
\hline Rigidez (N/mm) & PlaCF & 50 & 4 & \multirow{2}{*}{$<0.05$} \\
\cline { 2 - 4 } & ParCF & 69 & 11 & \\
\hline
\end{tabular}

No presente estudo, a resistência máxima à carga foi similar nos dois grupos experimentais, porém, a rigidez foi significativamente diferente entre os dois grupos. Zhang ${ }^{9}$ comparou as duas técnicas e demonstrou não haver diferença na qualidade da redução obtida e no resultado funcional dos pacientes. Embora o grupo com parafusos iliossacrais tenha tido menor sangramento operatório e menor tempo cirúrgico, ele foi mais dependente de imagens radiológicas intraoperatórias e levou a um maior número de complicações neurológicas. Elzohairy e Salama ${ }^{19}$ dividiram o tratamento das lesões instáveis da pelve em dois grupos: redução aberta e fixação interna da articulação sacroilíaca e redução fechada com fixação percutânea da articulação sacroilíaca com parafuso iliossacral. Não houve diferença nos resultados funcionais obtidos. Esse autor concluiu que a escolha da técnica a ser utilizada deve ser multifatorial, levando em consideração o tempo decorrido da lesão, condições gerais do paciente, condições de pele, experiência do cirurgião e presença de dismorfismo sacral. Diferentemente dos estudos supracitados, este estudo constituiu-se em um teste biomecânico in vitro, no qual mediu-se a carga suportada e rigidez das montagens. A redução foi sempre perfeitamente anatômica em função da visão direta da redução e da preservação da anatomia da amostra, o que sugere que a diferença entre a rigidez dos dois modelos poderia ser ainda maior na prática. A comparação de nossos resultados com os dos estudos de Zhang $^{9}$ e Elzohary e Salama ${ }^{19}$ sugere que a rigidez, pelo menos dentro da faixa de valores encontrados neste trabalho, pode ter pouca importância para o resultado funcional dos pacientes.

Além desses dois trabalhos citados acima, foram encontradas na literatura outras comparações diretas entre esses dois tipos de fixação. A falta de estudos que comparem em um mesmo modelo a resistência e a rigidez da fixação da luxação sacroilíaca com placas anteriores e parafusos iliossacrais apoia a apresentação do presente trabalho. Optou-se neste estudo por utilizar dois parafusos iliossacrais em S1, uma vez que estudos prévios $^{20,21}$ demostraram que o tratamento da luxação sacroilíaca com dois parafusos é superior ao tratamento com um parafuso, independente do local (S1 ou S2) de fixação. No outro grupo, optou-se por placas com três orifícios, pois segundo Chen, ${ }^{8}$ este tipo de placa oferece fixação mais resistente do que a placa com dois orifícios, principalmente se são colocadas em angulo de $60^{\circ}$ entre elas. Especial cuidado foi tomado na montagem dos modelos, pois Bai et al. ${ }^{22}$ demonstraram a importância da colocação correta das placas visando maximizar a estabilidade da fixação. As técnicas de fixação de ambos os estudos acima foram similares às aqui utilizadas. Ainda segundo Bai, ao fixar a articulação sacroilíaca com duas placas anteriores, uma das placas deve ser colocada no terço superior e a outra no terço médio da articulação. A margem de segurança para evitar lesão principalmente à raiz de L5 deve ser no máximo de $2.5 \mathrm{~cm}$ e $1.5 \mathrm{~cm}$ medialmente à articulação nos terços superior e médio da articulação sacroilíaca, respectivamente. Não devem ser colocadas placas no terço articular inferior. Levando em consideração essa pequena margem de segurança para fixação com placas, foi usada neste estudo dupla placa DC $3.5 \mathrm{~mm}$ em detrimento a placas mais largas $(4.5 \mathrm{~mm})$. 


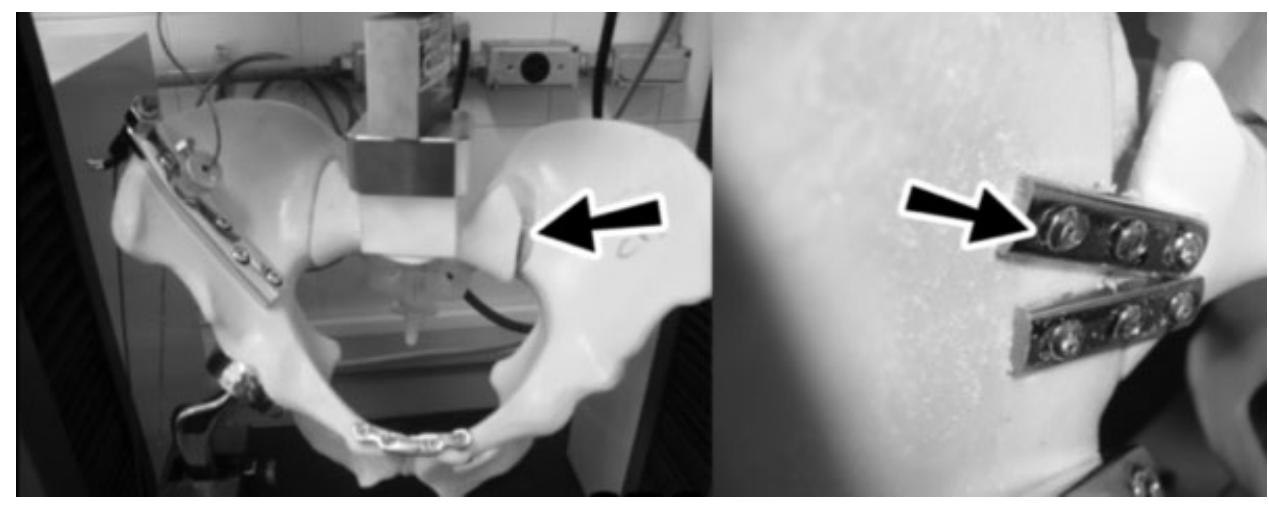

Fig. 7 Mecanismo de falha dos modelos testados (seta preta). No grupo ParCF (à esquerda) o mecanismo de falha ocorreu na articulação sacroilíaca contralateral. No grupo PlaCF (à direita) houve arrancamento dos parafusos da placa (no ilíaco) na articulação sacroilíaca testada. (fonte: arquivo pessoal).

Sagi et al. ${ }^{23}$ em estudo biomecânico, utilizaram três tipos de fixação da articulação sacroilíaca em lesões verticalmente instáveis (com sínfise aberta): um parafuso em S1, dois parafusos em S1 e um parafuso em S1 e outro em S2. Ambas foram testadas com e sem fixação da sínfise pubiana. Os resultados demonstraram que a fixação da sínfise pubiana aumentou de forma considerável a estabilidade das montagens, diminuindo significativamente desvios no plano axial, anteroposterior e lateral. No presente estudo, de acordo com a literatura, ${ }^{23,24} \mathrm{a}$ sínfise pubiana foi fixada nos modelos testados.

A utilização da fixação lombopélvica no tratamento das luxações sacroilíacas também é citada na literatura. Sobhan et al. ${ }^{25}$ apresentaram bons resultados com este método de tratamento, possibilitando carga precoce nos pacientes operados. Em nossa opinião sua melhor indicação seria em lesões pélvicas envelhecidas ou em dissociações espinopélvicas. Complicações de pele e dificuldade de colocação do paciente politraumatizados em decúbito ventral, dificultam a utilização desta técnica em casos agudos.

Nossos resultados demonstraram não haver diferença estatística em relação à carga máxima suportada pelos dois grupos testados, no tratamento da luxação sacroilíaca. Em relação à rigidez, o grupo que utilizou dois parafusos iliossacrais mostrou-se superior. Vale salientar que os mecanismos de falha foram diferentes em cada grupo testado.

Tais resultados apresentados devem estimular novos estudos com o objetivo de avaliar se a maior rigidez obtida com os parafusos iliossacrais pode ter alguma repercussão clínica em relação a carga mais precoce e a redução da dor no pósoperatório imediato. $O$ fato de não haver diferença significativa entre as cargas máximas suportadas por ambas as fixações sugere que a fixação com duas placas anteriores à articulação sacroilíaca é uma opção aceitável no tratamento das luxações sacroilíacas, na impossibilidade de utilizar parafusos iliossacrais.

Um fator limitante do estudo é o fato de terem sido usadas placas de $3.5 \mathrm{~mm}$ convencionais. Estudos com placas mais largas ou bloqueadas devem ser estimulados. Outra limitação deste estudo é o fato de terem sido utilizados modelos sintéticos. Estes têm características diferentes dos modelos cadavéricos em virtude da ausência de estruturas musculo ligamentares. Para que este fator de confundimento fosse reduzido ao máximo, foi usado um modelo de ensaio que pudesse reproduzir o apoio do membro durante a marcha associado a um sistema de cabos de aço para mimetizar em parte a ação das estruturas musculo ligamentares e assim impedir a rotação da hemipelve contralateral durante os testes. Por outro lado, embora as pelves sintéticas utilizadas não reproduzissem as propriedades mecânicas do osso humano, o objetivo do trabalho foi comparar os valores relativos obtidos para diferentes fixações da luxação sacroilíaca. A intenção era descobrir se uma fixação era mais rígida ou resistente que a outra, independentemente dos valores absolutos de rigidez e resistência. Assim, o uso de pelves sintéticas pode ter como vantagem em relação às pelves cadavéricas a diminuição da dispersão dos valores obtidos durante os ensaios. Como os modelos sintéticos foram do mesmo lote, houve uma capacidade maior de controle dos testes. Os modelos cadavéricos nem sempre apresentam a mesma característica, pois além da variação anatômica entre eles, muitas vezes não se sabe a idade, sexo, existência de doenças prévias e grau de desmineralização. ${ }^{26}$

\section{Conclusão}

Não há diferença em termos de carga máxima suportada, entre duas placas anteriores e dois parafusos iliossacrais na fixação da luxação da articulação sacroilíaca. No entanto, dois parafusos iliossacrais se mostraram uma fixação mais rígida quanto comparada com duas placas anteriores. É importante citar que os mecanismos de falha foram diferentes nos diferentes grupos testados.

Conflitos de Interesses

Os autores declaram não haver conflitos de interesses.

\section{Referências}

1 Kokubo Y, Oki H, Sugita D, et al. Functional outcome of patients with unstable pelvic ring fracture. JOrthop Surg (Hong Kong) 2017;25(01):2309499016684322

2 Dienstknecht T, Pfeifer R, Horst K, et al. The long-term clinical outcome after pelvic ring injuries. Bone Joint J 2013;95-B(04): 548-553 
3 Liu Y, Wang J, Zhang Y. Occult external iliac vein injury after anterior dislocation of the sacroiliac joint in adult patient. Acta Orthop Traumatol Turc 2017;51(02):169-171

4 Harvey-Kelly KF, Kanakaris NK, Obakponovwe O, West RM, Giannoudis PV. Quality of life and sexual function after traumatic pelvic fracture. JOrthop Trauma 2014;28(01):28-35

5 Zhang L, Peng Y, Du C, Tang P. Biomechanical study of four kinds of percutaneous screw fixation in two types of unilateral sacroiliac joint dislocation: a finite element analysis. Injury 2014;45(12): 2055-2059

6 Giráldez-Sánchez MA, Lázaro-Gonzálvez Á, Martínez-Reina J, et al. Percutaneous iliosacral fixation in external rotational pelvic fractures. A biomechanical analysis. Injury 2015;46(02):327-332

7 Miller AN, Routt ML Jr. Variations in sacral morphology and implications for iliosacral screw fixation. JAm Acad Orthop Surg 2012;20(01):8-16

8 Chen W, Pan ZJ, Chen JS. Biomechanical research on anterior double-plate fixation for vertically unstable sacroiliac dislocations. Orthop Surg 2009;1(02):127-131

9 Zhang R, Yin Y, Li S, Hou Z, Jin L, Zhang Y. Percutaneous sacroiliac screw versus anterior plating for sacroiliac joint disruption: A retrospective cohort study. Int J Surg 2018;50(01):11-16

10 Simpson LA, Waddell JP, Leighton RK, Kellam JF, Tile M. Anterior approach and stabilization of the disrupted sacroiliac joint. J Trauma 1987;27(12):1332-1339

11 Routt ML Jr, Meier MC, Kregor PJ, Mayo KA. Percutaneous iliosacral screws with the patient supine technique. Oper Tech Orthop 1993;3:35-45

12 Dienstknecht $\mathrm{T}$, Berner A, Lenich A, et al. Biomechanical analysis of a transiliac internal fixator. Int Orthop 2011;35(12): 1863-1868

13 Mullis BH, Sagi HC. Minimum 1-year follow-up for patients with vertical shear sacroiliac joint dislocations treated with iliosacral screws: does joint ankylosis or anatomic reduction contribute to functional outcome? J Orthop Trauma 2008;22(05):293-298

14 Dujardin FH, Hossenbaccus M, Duparc F, Biga N, Thomine JM. Long-term functional prognosis of posterior injuries in highenergy pelvic disruption. JOrthop Trauma 1998;12(03):145-150 , discussion $150-151$
15 Wu T, Chen W, Zhang Q et al. Biomechanical Comparison of Two Kinds of Internal Fixation in a Type C Zone II Pelvic Fracture Model. Chin Med J (Engl) 2015;128(17):2312-2317

16 von Keudell A, Tobert D, Rodriguez EK. Percutaneous fixation in pelvic and acetabular fractures: understanding evolving indication and contra- indications. Oper Tech Orthop 2015;25:248-255

17 Alla SR, Roberts CS, Ojike NI. Vascular risk reduction during anterior surgical approach sacroiliac joint plating. Injury 2013; 44(02):175-177

18 Elmanawy M, Elshoura S, Youssef S, Salama F. Treatment of sacroiliac joint disruption with anterior stabilization. Egypt Orthop J 2015;50(01):45-50

19 Elzohairy MM, Salama AM. Open reduction internal fixation versus percutaneous iliosacral screw fixation for unstable posterior pelvic ring disruptions. Orthop Traumatol Surg Res 2017;103 (02):223-227

20 van Zwienen CMA, van den Bosch EW, Snijders CJ, Kleinrensink GJ, van Vugt $A B$. Biomechanical comparison of sacroiliac screw techniques for unstable pelvic ring fractures. JOrthop Trauma 2004;18(09):589-595

21 Vigdorchik JM, Jin X, Sethi A, et al. A biomechanical study of standard posterior pelvic ring fixation versus a posterior pedicle screw construct. Injury 2015;46(08):1491-1496

22 Bai Z, Gao S, Liu J, Liang A, Yu W. Anatomical evidence for the anterior plate fixation of sacroiliac joint. JOrthop Sci 2018;23 (01):132-136

23 Sagi HC, Ordway NR, DiPasquale T. Biomechanical analysis of fixation for vertically unstable sacroiliac dislocations with iliosacral screws and symphyseal plating. JOrthop Trauma 2004;18 (03):138-143

24 Varga E, Hearn T, Powell J, Tile M. Effects of method of internal fixation of symphyseal disruptions on stability of the pelvic ring. Injury 1995;26(02):75-80

25 Sobhan MR, Abrisham SM, Vakili M, Shirdel S. Spinopelvic fixation of sacroiliac joint fractures and fracture- dislocations: A clinical 8 years follow- up study. Arch Bone Jt Surg 2016;4(04):381-386

26 van Arkel RJ, Jeffers JRT. In vitro hip testing in the International Society of Biomechanics coordinate system. JBiomech 2016;49 (16):4154-4158 